\title{
Miniature Mobile Sensor Platforms for Condition Monitoring of Structures
}

\author{
Markus Friedrich $^{* 1}$, Member, IEEE, Gordon Dobie ${ }^{* 1}$, Student Member, IEEE, Chung Chee Chan ${ }^{* 1}$, S. Gareth \\ Pierce $^{* 1}$, Walter Galbraith ${ }^{* 1}$, Stephen Marshall, Member ${ }^{* 2}$, IEEE, Gordon Hayward ${ }^{* 1}$, Fellow, IEEE.
}

\begin{abstract}
An autonomous, multi-sensor inspection system for non-destructive evaluation (NDE) of ferromagnetic materials is described. The sensor configuration enables two inspection modes - magnetic (flux leakage and eddy current) and non-contact ultrasound. Each is designed to function in a complementary manner, maximizing the potential for detection of both surface and internal defects. Particular emphasis is placed on the generic architecture of a novel, intelligent sensor platform and its positioning on the structure under test. The sensor units are capable of wireless communication with a remote host computer, which controls manipulation and data interpretation. Results are presented in the form of autonomous scans with different NDE sensors in a series of experiments on thin plate structures. To highlight the advantage of utilizing multiple inspection modalities, data fusion approaches are employed to combine data collected by complementary sensor systems. Fusion of data is shown to demonstrate the potential for improved inspection reliability.
\end{abstract}

\section{Index Terms - robotics, NDE, sensor platforms}

\section{INTRODUCTION}

Condition monitoring of structures is becoming increasingly important as society attempts to deal with ageing civil infrastructure, transportation systems and industrial plant. In many instances manual inspection is problematic, perhaps due to health and safety risks, or is too time consuming, with the attendant cost implications. As a result, robotic systems are gaining increasing attention for structural NDE, although to date these have been confined mainly to relatively large, single issue inspection processes devoted to performing a specific inspection task. Recently, there has been considerable interest towards miniaturization and the application of distributed autonomous micro systems, containing increasingly small and more adaptable robotic technology. One example involves a fleet of miniature vehicles, each capable of independent communication and tasking under the control of a host system. Such multiple cooperative vehicles can exploit information derived from a variety of disparate sources, simultaneously performing tasks faster and more efficiently than a single inspection robot and, with appropriate intelligence, can compensate for individual failures. Due to their miniature size, access problems are reduced and since individual robots are expendable, only limited capabilities are lost in the event of malfunction of a single unit. The major requirements of these systems are cost effectiveness, reliability, mobility and complete autonomy. To date, a wide range of research involving robotic vehicles for NDE has been reported [1], [2]. This includes teams of small robots for reconnaissance and surveillance [3], tethered large scale inspection robots for hulls of vessels [4], pipes [5], and storage tanks [6]. Separate developments that involve concepts of collaborating robots [7] and climbing techniques for small mobile robots for different surfaces and surface conditions and with low power consumption have been presented [8], [9]. The problem of total surface coverage, a characteristic task for mobile robots in for example, lawn mowing and floor cleaning, has been investigated [10].

This paper describes the adaptation of these techniques to create a novel prototype inspection system that is based on a mobile, multiple sensor platform, in the form of a miniature, autonomous robotic vehicle. Each platform is designed to adhere to inclined and curved ferromagnetic surfaces and is able to perform different inspection tasks with different sensor payloads. For such applications it is essential that the sensing technologies are both complementary and are independent of the need to maintain consistent contact with the surface of the test specimen. Magnetic techniques, for example the use of eddy currents, are suited to non-contact detection of near surface defects. However, they are generally unsuitable for volumetric scanning of the internal structure. Ultrasonic methods, in contrast, are able to perform scanning of the internal structure, but normally require some form of liquid or gel based material to couple the ultrasonic energy from the transducer into the test material. Provision and consistency of adequate coupling can be problematic for a small vehicle, with limited power supply capability. The current sensor platform comprises a magnetic flux leakage (MFL) system, combined with an eddy current (EC) unit and an air coupled ultrasonic (US) sensor arrangement. All are potentially non-contact and have been configured to minimize electrical power consumption. Results are presented that have been obtained by

Manuscript submitted $29^{\text {th }}$ September 2008

*1 Centre for Ultrasonic Engineering, University of Strathclyde, Royal College Building, 204 George Street, Glasgow, G1 1XW

*2 Centre for Signal and Image Processing, University of Strathclyde, Royal College Building, 204 George Street, Glasgow, G1 1XW 
automatic scans in a series of experiments on test plates. The different data sets obtained from such scans were combined using data fusion methods based on probabilistic and evidence based approaches (Bayesian and Dempster-Shafer). The fusion of magnetic and ultrasonic scan data is demonstrated and the constraints imposed by the ultrasonic positioning system, used for determination of vehicle location in the experimental scans, are discussed in detail.

\section{SYSTEM DESIGN}

The major technology challenges in the design of a prototype sensor platform are power handling, positioning, dexterity, cost effectiveness and climbing skills, in order to cope with various types of inclined and curved surfaces and ceilings. The sensor architecture was based on reconfigurable modules as highlighted on the prototype shown in Figure 1. This allows for modification and provides the capacity for inspecting various test objects, materials and surface conditions by carrying a range of NDE inspection tools. For traction on ferromagnetic surfaces, permanent magnetic wheels were selected. These not only provided adequate grip on all of the test surfaces but also created a magnetic field within the test specimen, between the wheels, facilitating the implementation of MFL, as described in Section III.B. Several robotic sensor platforms were assembled and tested with different NDE payloads under laboratory conditions.

The robotic software executes on two interacting systems, comprising an on-board microcontroller for analysis of sensor data and low-level control functions, combined with a portable host PC that handles absolute positioning, data fusion, high level planning, decision making and coordination.

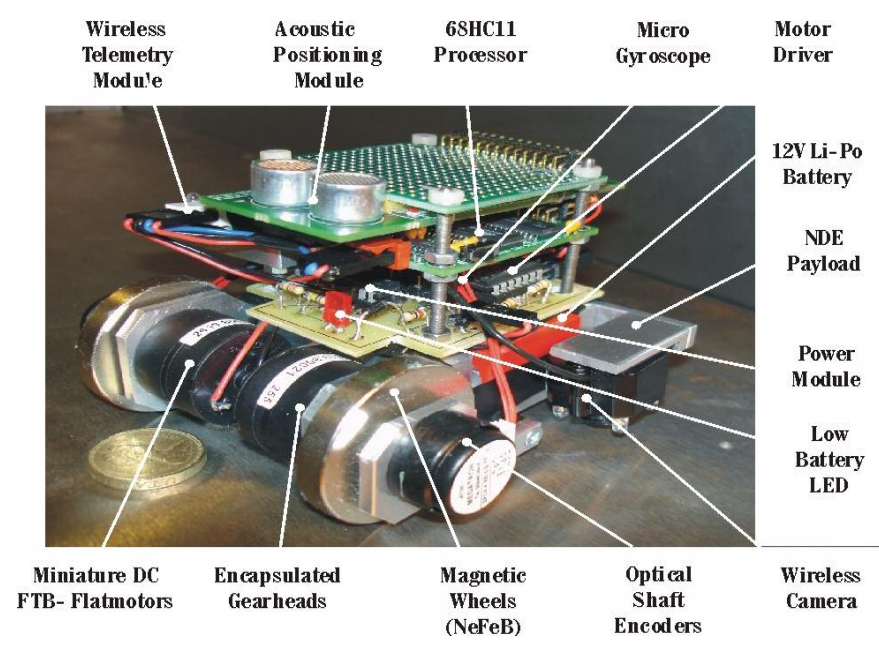

Figure 1: Prototype Mobile Inspection Unit

As accurate straight line movements and constant velocity are crucial for most inspection tasks, a low level PI-control loop operates locally on each sensor platform, relying solely on odometric data. This reference based positioning system was calibrated via routing based on the UMBmark experiment described in [11] and allows for sufficient autonomy of the individual units. The odometric positioning was complemented by a local absolute beacon-listener system: Low cost Cricket units (Crossbow) [12] were configured as fixed passive listeners, mounted on a $2 \times 2 \mathrm{~m}$ portable frame, and as active beacons on the platforms. Absolute 3D position information was obtained by trilateration [13]. To obtain the distances between any vehicle and the three listeners, each beacon periodically broadcasts its space identifier on an RF channel and isochronously, an ultrasonic pulse. The listeners receive both pulses with a time difference from which the distance between one listener and a beacon is calculated. The Cricket system has a range of 10.5 meters, requiring line of sight [12] between each platform and the beacon system.

The variance of each distance measurement for trilateration is $1 \mathrm{~cm} / \mathrm{m}$. According to Gauss' law of error propagation, for a measurement result $y=f(A, B, C, \ldots)$ derived from different independent measurands $\mathrm{A}, \mathrm{B}$ and $\mathrm{C}$, the resulting measurement uncertainty, $\mathrm{u}(\mathrm{y})$, is dependent on the measurement uncertainties of the individual measurands $u(A)$, $\mathrm{u}(\mathrm{B})$ and $\mathrm{u}(\mathrm{C})$ and is given by Eq. (1).

$$
u(y)=\sqrt{\left(\frac{\partial f}{\partial A} u(A)\right)^{2}+\left(\frac{\partial f}{\partial B} u(B)\right)^{2}+\left(\frac{\partial f}{\partial C} u(C)\right)^{2}+\ldots}
$$

Eqn. 1

This yields the following uncertainties in the three positional estimate components:

$$
\begin{gathered}
u\left(x_{r}\right)=\sqrt{\left(\frac{d_{1}}{r} u\left(d_{1}\right)\right)+\left(-\frac{d_{2}}{r} u\left(d_{2}\right)\right)} \\
u\left(y_{r}\right)=\sqrt{\left(\frac{d_{1}}{r} u\left(d_{1}\right)\right)+\left(-\frac{d_{3}}{r} u\left(d_{3}\right)\right)} \\
u\left(z_{r}\right)=\sqrt{\left(\frac{\partial z_{r}}{\partial d_{1}} u\left(d_{1}\right)\right)^{2}+\left(\frac{\partial z_{r}}{\partial d_{2}} u\left(d_{2}\right)\right)^{2}+\left(\frac{\partial z_{r}}{\partial d_{3}} u\left(d_{3}\right)\right)^{2}}
\end{gathered}
$$

Eqn. 3

Eqn. 4

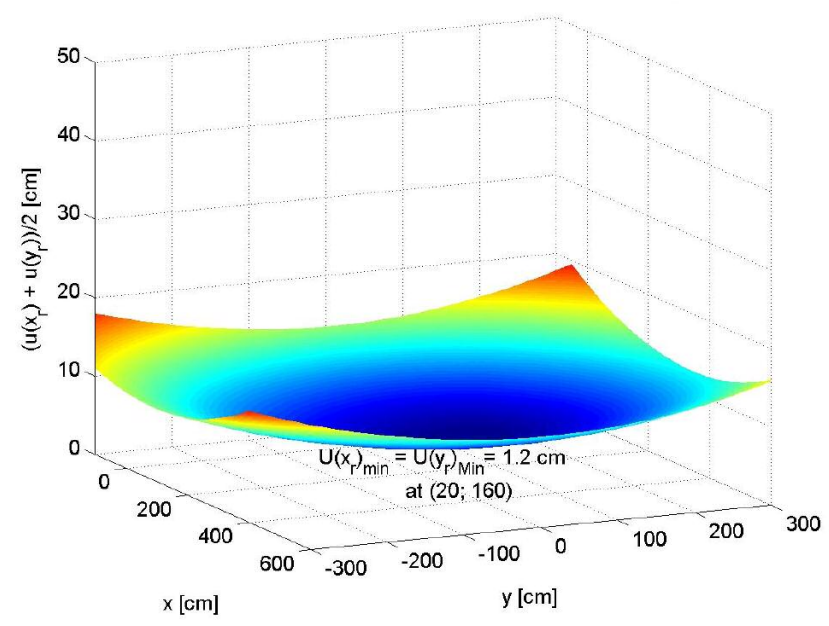

Figure 2: Propagated uncertainties for $\mathrm{x}$ and $\mathrm{y}$ following Gauss' law of error propagation. Frame distance from surface $=50 \mathrm{~cm}$, inter-listener distance $=300 \mathrm{~cm}$

For a plane parallel to the plane defined by the portable frame, this yields the theoretical average positioning accuracy in ' $\mathrm{x}$ ' and ' $y$ ' shown in Figure 2. Highest accuracy $(\mathrm{u}(\mathrm{xr}) \mathrm{min}=$ $\mathrm{u}(\mathrm{yr}) \min =1.2 \mathrm{~cm})$ is at $\mathrm{P} 0=[20 ; 160]$, in the central area between listeners on the frame in the first quadrant. This result was validated experimentally by positioning the beacon at 
different known positions in 3D space. As the error increases further away from $\mathrm{P} 0$, position and orientation update of each platform takes place within a circular area $\mathrm{A} 0,50 \mathrm{~cm}$ around P0. By decreasing the inter-listener distance the accuracy can be improved, but this reduces the work area.

Further improvement of the tracking task utilizing the distance measurements of the absolute positioning system was achieved by implementing a weighted median filter for outlier rejection in combination with a Kalman Filter [14]. The resulting performance of the implemented filter is shown in Figure 3.

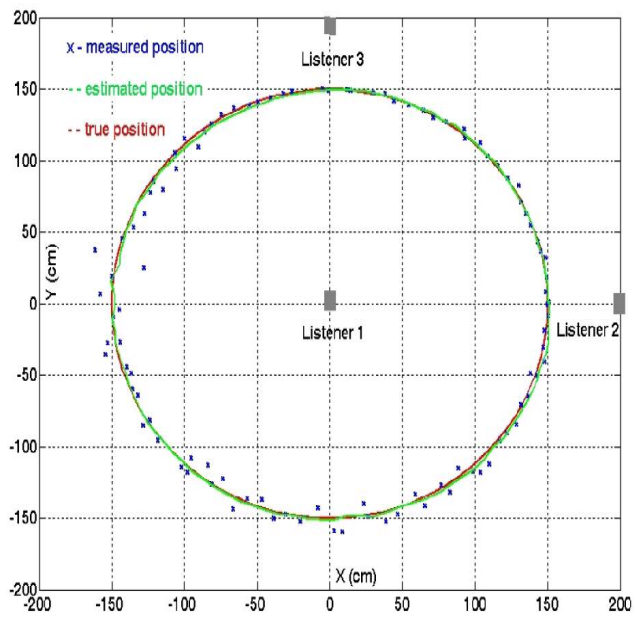

Figure 3: Performance of the Kalman filter on a simulated circular path.

The dashed line represents the simulated circular path of the inspection vehicle, the separate crosses represent the noise affected measurements of the beacon system and the path with the applied Kalman filter is plotted as a continuous line. Clearly visible is the reduced accuracy in the third quadrant resulting from larger variances of the distance measurement for greater beacon-listener distances as described previously.

\section{THE INSPECTION SENSOR UNITS}

\section{A. Eddy Current Inspection}

For testing of electrically conducting materials, EC techniques are widely accepted for reliable NDE and are particularly effective for investigating service induced fatigue and stress corrosion cracks [15], [16]. EC testing is based on alternating currents in a coil, which induce eddy currents in an electrically conducting test material, when the coil is placed in close proximity to the material under test. The eddy currents generate a reverse magnetic field, which affects the loading on the coil and changes its impedance. A discontinuity within the material, such as a fatigue crack positioned underneath the test coil, interrupts or reduces the eddy current flow, thus decreasing the loading on the coil and increasing its effective impedance. This form of sensor is restricted to electrically conducting test materials and is best suited for the detection of surface breaking defects. It can thus be considered as being complementary to the ultrasonic sensor unit described in Section III.C.

To compensate for changes in material properties and temperature, a differential probe was utilized, where two coils form the branches of a bridge circuit. Any change in the condition of either coil results in an unbalanced bridge, with the degree of imbalance corresponding to the change in coil impedance and provides an indication of defect presence. Two coils, each $1 \mathrm{~mm}$ in diameter, were positioned on a vertically adjustable spring loaded lever. This arrangement served to maintain constant sensor position by holding the probe in a fixed location relative to the surface, without inhibiting motion of the platform. At a working frequency of $180 \mathrm{kHz}$, reliable discrimination between impedance changes that result from variations in lift-off and those that are caused by the presence of a defective region is possible. Provided that the probe is orientated so that the connecting line of the centre of the two coils is parallel to the main driving direction of the platform, the front coil passes a crack or an indentation before the rear coil, and the algebraic sign of the phase change gives a clear indication of the type of irregularity. After calibration, a software routine that utilizes averaged gradients was employed to aid identification of surface breaking defects during the sensor scanning operations described in Section IV.

\section{B. Magnetic Flux Leakage Inspection}

MFL is a commonly used NDE technique for ferromagnetic materials and is one of the most economical methods for detection of material loss due to corrosion, pitting or gouging [6], [17], [18]. It finds widespread application for in-service inspection of oil and gas pipelines, wire ropes, and floors of storage tanks, where corrosion pits can develop at a significant rate, as stated previously [17], [19]. Usually, permanent or electromagnets are used to magnetize the test surface to near saturation flux density and under normal conditions, the magnetic lines of force, are predominantly contained inside the ferromagnetic material [20]. However, if there is a surfacebreaking or near-surface flaw, there is a local change in permeability, and the field is distorted, causing local magnetic flux leakage. A magnetic field detector is placed between the poles of the magnet to detect the leakage field, identify damaged areas and estimate the depth of metal loss.

In the present platform design, traction is provided by two permanent magnet wheels as described previously. The region of test material between the wheels is thus subject to magnetization and this presents a convenient opportunity to detect any leakage flux arising from surface of near-surface defects in this region. The commercial finite element code Comsol [21] was used initially to evaluate feasibility and successful experiments were conducted using a linear Hall Effect sensor (Honeywell SS94A2), combined with the induced field of the platform wheels. The method was then refined to extend coverage and reliability by incorporation of a 3 element linear array of Hall sensors, located between the wheels. The array is mounted on a spring loaded lever to maintain constant sensor lift-off and is orientated along the imaginary connection line between the two contact points of the magnetic wheels. Two $25 \mathrm{~mm}$ shoulders at the bottom side 
of the mounting platform guarantee a lift-off of $0.25 \mathrm{~mm}$ of the $1 \mathrm{~mm}$ thick Hall elements and prevent damage while the elements travel along the surface.

As in the case of the EC-sensor, a software routine that utilises averaged gradients over a number of measurements was used to improve actual flaw recognition. The sensitivity is adjustable by calibration and allows for operation on different materials and at different inspection velocities. In this way, changing the threshold affects the number of False Positives and False Negative decisions for a particular defect.

\section{Ultrasonic Inspection}

Ultrasound affords additional flexibility for condition monitoring and NDE. Ultrasonic transducers and arrays can interrogate the interior volume of the structure under test and moreover, the technology is amenable to large area scanning, an important issue for cost effective monitoring of larger structures. However, as stated previously, conventional ultrasonic inspection requires the provision of a suitable coupling liquid between the probe and the structure under test. Maintaining the supply and consistency of coupling is problematic for the type of mobile system under consideration and this is also compounded by the need to minimize the size of the sensor platforms.

An alternative approach is to use air as the coupling fluid and through optimization of the transducer and associated electronics, compensate for the inevitable loss of sensitivity, which can be as much as $30 \mathrm{~dB}$ when compared with a conventional liquid coupled system. However, the operating frequency is usually restricted to below $1 \mathrm{MHz}$, due to the high attenuation in air and accordingly, resolution is reduced due to the longer wavelengths in the test material. However, previous work by some of the authors and others, [22], [23] has shown the validity of through air, Lamb wave ultrasonic inspection. Here a pair of air coupled ultrasonic transducers were inclined at an appropriate angle to the test specimen, to generate the zeroth order anti-symmetric $\left(\mathrm{A}_{0}\right)$ Lamb wave in both plate and curved metallic structures. The $\mathrm{A}_{0}$ Lamb wave mode is dispersive, with the phase velocity dependent on the material properties and the frequency thickness product. A change in plate thickness, due to erosion or otherwise, results in attenuation of the Lamb wave signal at the receiving transducer and it is by this mechanism that defects are detected. Crack type defects may also scatter the Lamb wave further attenuating the received signal. In this earlier work, relatively narrowband 1-3 piezoelectric composite transducers were found to be the most sensitive form of ultrasonic generation and sensing device, when interfaced to customized ultrasonic matching layers [24], electrical matching circuitry and extremely low noise amplification [22]. However, the systems described in [22], [23] utilized high excitation voltages, of several hundred volts, in a tone burst mode, rendering them impractical for the small, autonomous vehicles under consideration.

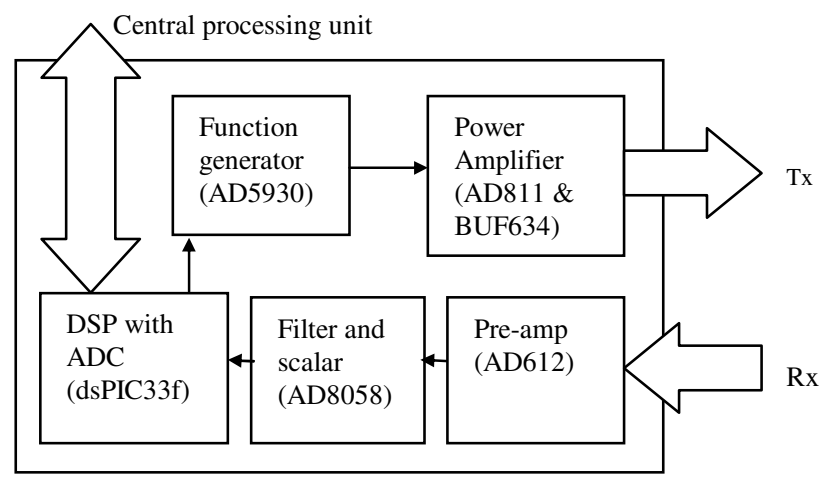

Figure 4: Block Diagram of embedded ultrasonic payload circuitry.

Experiments were conducted to assess the feasibility of this approach, using an air-coupled payload that was powered entirely from the onboard battery and was compact enough to be carried by the sensor platform. A pair of piezoelectric composite transducers were constructed according to the methods described in [22], [23]. For testing purposes, the pair was mounted on the platform and inclined at an angle of approximately $10^{\circ}$, to generate and receive the $\mathrm{A}_{0}$ mode at 600 $\mathrm{kHz}$ in a $1.5 \mathrm{~mm}$ thick aluminum plate. The drive signals were generated by an onboard, customized waveform generator and power amplifier configuration and the received signal captured digitally for local processing or wireless transmission to the host PC. Figure 4 shows a block diagram of the embedded electronics with the principal components for each block in brackets. The drive electronics can generate a $\pm 34 \mathrm{~V}$ sine wave drive signal used to excite the composite transmitting device. The frequency and packet length of both signals are configurable in software, so can be set to match different transducer devices. The receiver uses a low noise preamplifier that interfaces with the receiving transducer. The output of the preamplifier is band-pass filtered in hardware and then level shifted and fed into an analogue to digital converter (ADC). The digitized signal is sent by serial link to the central processing unit. 

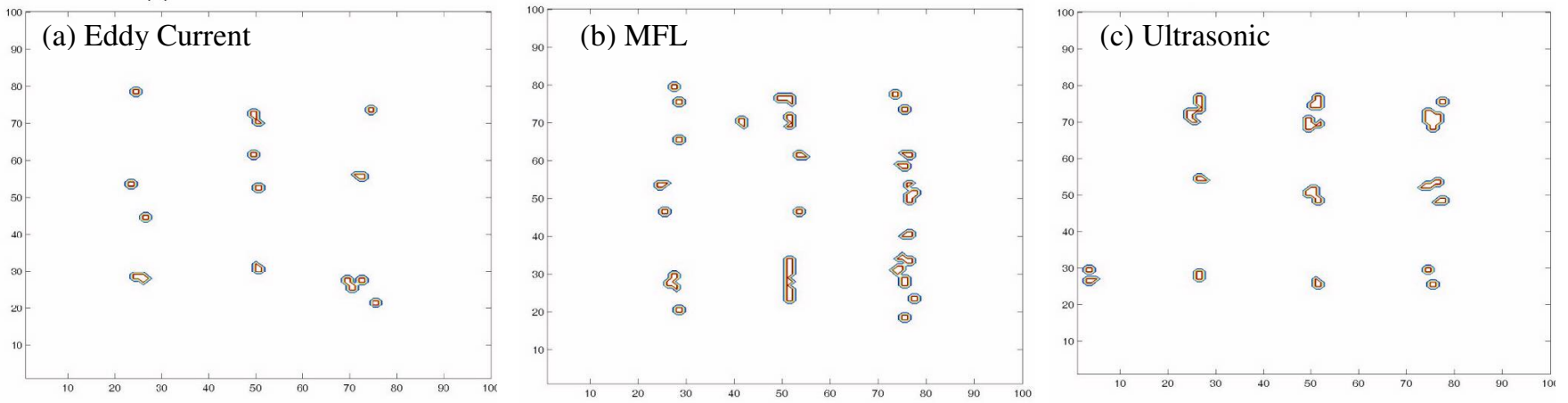

Figure 6. Contour map representations of scan results: (1) Eddy Current results, steel plate, (b) Magnetic Flux Leakage results, steel plate, (c) Ultrasonic Inspection results, aluminum plate

\section{EXPERIMENTAL TESTING AND DATA FUSION}

The experiment involved two $1 \mathrm{~m} \times 1 \mathrm{~m}$ plates of mild steel and aluminum, which contained a set of nine $3 \times 1 \mathrm{~cm}$ artificial defects, varying in depth from $10 \%$ to $90 \%$ of total plate thickness and distributed equally with a spacing of $25 \mathrm{~cm}$, as shown in Figure 5. Overall thickness of both plates was 1.5 $\mathrm{mm}$. To achieve complete coverage of the inspection area, the sensor platform performs a raster scan. Due to its simple geometry, the specimen can be decomposed into rectangular cells for simultaneous inspection with multiple vehicles to accelerate the process of coverage. However, if the vehicles carry different NDE sensors, the same cells have to be covered repeatedly. The path for each vehicle was calculated off line and controlled by the host PC. Together with the absolute positioning information this data was used to construct $\mathrm{C}$-scan images using EC, MFL and US sensors.

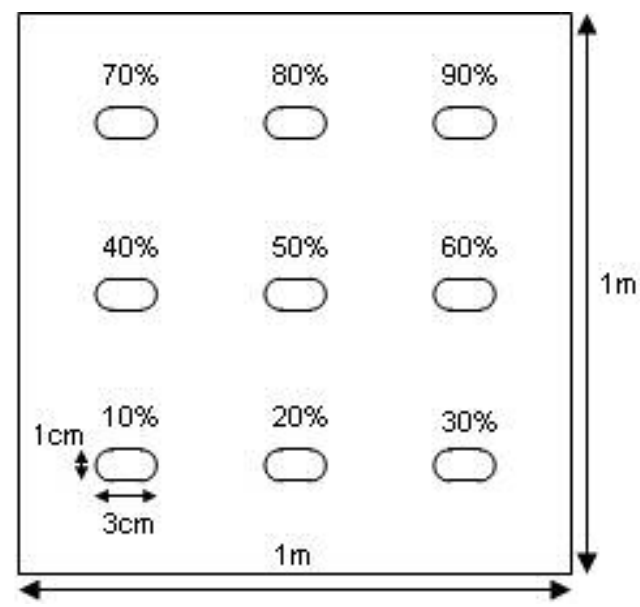

Figure 5: Distribution of defects on test specimen

Under specified inspection conditions and procedures and for a defect of a given type and size, repeated inspections do not necessarily result in consistent hit or miss indications. In fact, the spread of detection results for each defect size and type is represented by the quantitative statistical parameter probability of detection (PoD) [25]. For a specific test method and test protocol, there are four possible outcomes for an inspection of a component: True Positive (a defect indication is reported and there is an actual defect present), False Positive (a defect indication is reported where no actual defect exists), False Negative (no defect is reported and an actual defect is present) and True Negative (no defect is reported and no defect is present). Table 1 illustrates the conventional arrangement of these four possibilities into the probability confusion matrix of detection. In the following sections, the PoD parameter is used to quantify defect detection for the different NDE modalities employed by the mobile sensor platform.

\begin{tabular}{|c|c|c|c|}
\hline & & \multicolumn{2}{|c|}{ Actual class } \\
\hline & & Defect $(a=1)$ & No Defect $(a=0)$ \\
\hline \multirow{2}{*}{ 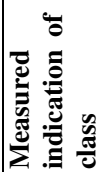 } & $\begin{array}{l}\text { Positive } \\
(b=1)\end{array}$ & $\begin{array}{l}\text { TRUE POSITIVE } \\
\text { RATE (TP) }\end{array}$ & $\begin{array}{l}\text { FALSE POSITIVE } \\
\text { RATE (FP) }\end{array}$ \\
\hline & $\begin{array}{l}\text { Negative } \\
(b=0)\end{array}$ & $\begin{array}{l}\text { FALSE NEGATIVE } \\
\text { RATE (FN) }\end{array}$ & $\begin{array}{l}\text { TRUE NEGATIVE } \\
\text { RATE (TN) }\end{array}$ \\
\hline
\end{tabular}

Table 1: Confusion matrix between actual and measured class membership

The PoD confusion matrices for these different sensor techniques are shown in Table 2. Note the exact PoD figures depended on where a programmable critical threshold was set. The resulting C-scan utilizing the EC sensor payload is illustrated in Figure 6(a). Based on the output signal of the middle element from the central MFL sensor, the C-scan image in Figure 6(b) of the $1 \mathrm{~m} \times 1 \mathrm{~m}$ steel plate was produced, permitting a comparison of the performance of the Hall array with the EC-payload. Essentially, both systems are passive and are inherently suited to detection of surface or near-surface flaws. Performance on the plate structures is expected to be similar and although this appears to be confirmed by comparison of Figures 6(a) and 6(b), the PoD data from the test scans indicated slightly superior performance from the EC system.

The EC sensor has the advantage that it can be used on any electrically conducting material, while the MFL system is confined to ferromagnetic materials. However, since the platform traction system is reliant on magnetic adhesion, a single pair of EC sensors does not confer significant additional advantage. However, area coverage can be increased by using an array of EC coils and this will be reported at a later date. 


\begin{tabular}{|l|l|}
\hline Method & \multicolumn{2}{|l|}{ Confusion Matrix } \\
\hline Eddy Current & {$\left[\begin{array}{ll}0.8 & 0.2 \\
0.2 & 0.8\end{array}\right]$} \\
\hline $\begin{array}{l}\text { Magnetic Flux } \\
\text { Leakage }\end{array}$ & {$\left[\begin{array}{ll}0.7 & 0.3 \\
0.3 & 0.7\end{array}\right]$} \\
\hline Ultrasonic & {$\left[\begin{array}{ll}0.9 & 0.1 \\
0.1 & 0.9\end{array}\right]$} \\
\hline
\end{tabular}

Table 2: Confusion matrices for different NDE payloads

Finally, the active ultrasonic Lamb wave system was tested on the rear side of the $1.5 \mathrm{~mm}$ thick aluminum plate with the externally driven transducers generating and receiving the A0 Lamb wave at a frequency of $600 \mathrm{kHz}$. The attenuation of the Lamb wave caused when the nine different defects are between the transmitting and receiving transducer is clearly visible in Figure 6(c). Interestingly, the additional 'defect' shown on the left hand side of Figure 6(c) was introduced by localized thinning of the plate and this was not detected by the EC or MFL methods.

\section{B. Data Fusion}

It is obvious from Figure 6, that the different sensor technologies provided different indications of damage on the sample plates. Care must be exercised in directly comparing such sensor data as it is clear that fundamentally different physical mechanisms may lie behind the interactions between defect and a particular NDE technique. The dangers of naïve multi-sensor fusion lie in situations where contra-indication is generated by different sensing approaches [26]. However in situations where the data sets are non contra-indicative, a variety of approaches for combining data sets are available including fuzzy logic [27], neural networks [28], Bayesian approaches [29] and Dempster-Shafer evidence based [30]. A review of such approaches specific to NDE applications is given by [31].

1) Bayesian Probabilistic Approach

The principle behind a Bayesian [32] approach is that repeated observations are used to update the probability distribution that a particular hypothesis may be true (for example presence of defect). An a-priori probability of a hypothesis or conditional probability is used to produce a posterior probability of this hypothesis, updated as new information is conveyed to the system with Bayes' update rule.

Given 'n' mutually exclusive hypothesises $\left(H_{i}\right)$ that an event $\mathrm{E}$ will occur, then the posterior probability of $H_{i}$ given that $E$ is true is:

$$
P\left(H_{i} \mid E\right)=\frac{P\left(E \mid H_{i}\right) P\left(H_{i}\right)}{P(E)}
$$

Eqn. 5

The first term of the numerator in Eq 5 is the conditional probability of $E$ given $H_{i}$, this is often referred to as the "likelihood". The second term of the numerator is the "prior" probability of $H_{i}$, where the probabilities of all hypotheses sum to unity. The denominator is referred to as the "evidence" or marginal likelihood or unconditional probability, i.e. the probability of $E$ under all possible conditions; as given in Equation 6.

$$
P(E)=\sum_{j} P\left(E \mid H_{j}\right) P\left(H_{j}\right) \quad \text { Eqn. } 6
$$

The attraction of the Bayesian Evidence technique is that the evaluated posterior probability can become the prior for the next iteration of Bayes' rule application. In this way it is possible to combine a number of sets of data in successive fashion, each data set bringing new evidence to support or refute previous data. A common source of contention in the Bayesian technique is in setting the value of the initial prior. However if one is only interested in the relative increase in the probability of a defect, then this is not critical, whereas if an absolute measure of defect probability were required then the value of the prior selected would be essential.

2) Dempster-Shafer Evidence Approach

Dempster-Shafer theory [33], [34], [35] is a generalisation of the Bayesian theory of subjective probability, with the advantage of being capable of handling uncertain, imprecise, and incomplete information. Dempster-Shafer theory is based on obtaining degrees of belief for one question from subjective probabilities for a related question and a rule for combining such degrees of belief with independent items of evidence. A Frame of Discernment $\Theta$, also referred to as Universe of Discourse, is defined initially:
$\Theta=\left\{H_{1}, H_{2}, \ldots, H_{n}\right\}$
Eqn. 7

This set of mutually exclusive and exhaustive alternatives defines the working space for the application being considered and consists of all propositions for which the information sources provide evidence. The evidence is expressed by (probability) mass-values $m$, which are subsets of $\Theta$ and have to satisfy the conditions:
$0 \leq m\left(A_{i}\right) \leq 1$
Eqn. 8
$m(\phi)=0$
Eqn. 9

where $m\left(A_{i}\right)$ represents the strength of a single hypothesis $H_{i}$ and $\varphi$ is the empty set.

The fundamental difference between evidential and probabilistic reasoning is that not all the probability mass need be exhaustively assigned to individual events, and it is through this mechanism that the theory can accommodate uncertainty in outcomes. The belief, plausibility and doubt functions in event $\mathrm{A}_{\mathrm{i}}$ are represented as follows:

$$
\operatorname{Bel}\left(A_{i}\right)=\sum_{A_{j} \subseteq A_{i}} m\left(A_{j}\right) \quad \text { Eqn. } 10
$$

$\operatorname{Pls}\left(A_{i}\right)=\sum_{A_{j} \cap A_{i} \neq 0} m\left(A_{j}\right)=1-\operatorname{Dou}\left(A_{i}\right) \quad$ Eqn. 11

$\operatorname{Dou}\left(A_{i}\right)=\operatorname{Bel}\left(\underline{\underline{A_{i}}}\right)$

Eqn. 12 
The Bel function represents the minimum uncertainty value around $A_{i}$, whilst the $P l s$ is a measure for the maximum uncertainty value about the hypothesis. Dou represents the negation of the proposition $A_{i}$. The double underline in Eqn. 12 indicates negation.

In order to combine different data sets it is important to consider Dempster's combination rule. A fundamental issue arises if the intersection between supported propositions $A_{i}$ and $A_{j}$ is the empty set. If this arises, then a non-zero mass may be assigned to the empty set in contradiction with the basic definitions. To prevent this situation, the sum of mass assignments with non overlapping propositions are re-scaled such that $\varphi=0$. A disadvantage of this normalization is that the effects of conflict are ignored by the assignment to the empty set, and this behavior can sometimes produce counterintuitive results [35].

The joint mass is given by:

$$
m_{12}\left(A_{i}\right)=\frac{1}{1-K} \sum_{A_{p} \cap A_{q}=A_{i}} m_{1}\left(A_{p}\right) m_{2}\left(A_{q}\right)
$$

where

$$
\begin{array}{ll}
K=\sum_{A_{p} \cap A_{q}=\phi} m_{1}\left(A_{p}\right) m_{2}\left(A_{q}\right) & \text { Eqn. } 14 \\
\text { 3) Application of Bayesian and } & \text { Dempster-Shafer } \\
\text { Techniques to multi-sensor data fusion } &
\end{array}
$$

Comparing the contour plots of different NDE payloads in figure 6 with the true defect positions (figure 5), it is clear that the measured defect locations deviate from the true (known) positions. To enable a comparative quantification of the accuracy of each NDE scan, the mean square sum of errors between the true and indicated defect centers were calculated according to equation 15 .

$$
M S E=\frac{1}{N} \sum_{i=i}^{N}\left(x_{i}-x_{d i}\right)^{2}+\left(y_{i}-y_{d i}\right)^{2} \quad \text { Eqn. } 15
$$

Where $\mathrm{N}$ is the number of defects ( $\mathrm{N}=9$ in this case), $\left(x_{i}, y_{i}\right)$ is the indicted center of defect from NDE scan and $\left(x_{i d}, y_{i d}\right)$ is the known center of defect. Table 3 lists the calculated mean square errors for each of the different NDE payloads.

\begin{tabular}{|l|l|}
\hline NDE Method & $\begin{array}{l}\text { Mean Square } \\
\text { Error }\left(\mathbf{m m}^{2}\right)\end{array}$ \\
\hline Eddy Current & 1620 \\
\hline Magnetic Flux Leakage & 1940 \\
\hline $\begin{array}{l}\text { Ultrasonic } \\
\text { (air coupled Lamb wave) }\end{array}$ & 1550 \\
\hline
\end{tabular}

Table 3. Mean square errors of measured defect positions using different NDE payloads

Applying Bayesian probabilistic fusion on the EC and MFL data for calculating the posterior probabilities of a pixel being defective, if one inspection method reported a defect, then the probability of an indicated defect for a real defect, $\left[P\left(E \mid H_{0}\right)\right]$, corresponded to the True Positive Rate of the confusion matrix. Similarly the probability of an indicated defect when no defect was present, $\left[P\left(E \mid H_{l}\right)\right]$, corresponds to the False Positive Rate. As the edge length of a pixel was $1 \mathrm{~cm}$, and there were nine $3 \mathrm{~cm} \times 1 \mathrm{~cm}$ defects in the $1 \mathrm{~m} \times 1 \mathrm{~m}$ test specimen, an a-priori probability of containing a defect of 0.0027 was attributed to each pixel, i.e. $P\left(H_{0}\right)=0.0027$. In other words, before inspection, each square centimetre pixel had a probability of $0.27 \%$ of containing a defect. Using the Bayesian update rule, if a defect was detected via EC inspection, then this probability increased to $1.07 \%$, while it decreased to $0.067 \%$ if the inspection yielded a negative result. These two values became the new a-priori probabilities for the subsequent iteration of Bayes' rule where the results of the MFL scan were taken into account. Depending on whether a defect was detected with this second method or not, the probability of a defect being present changed as illustrated in Figure 7(a). The red lines indicate areas with a probability of 9.1 $P\left(H_{0}\right)$, so these positions were 9.1 times more likely to contain a defect than positions which were not subject to inspection. The areas marked by green boundary lines have a posterior probability of containing a defect of $1.7 P\left(H_{0}\right)$ and for the areas within the blue lines $P\left(H_{0} \mid E\right)=0.6 P\left(H_{0}\right)$ after the second iteration step. In the remaining white areas, where both inspection techniques did not report a defect, the probability of a defect being present was 9.3 times lower than without inspection. The calculated mean square error between the indicated and known defect positions yielded a figure of 1220 $\mathrm{mm}^{2}$, an improvement over the values of 1620 and $1940 \mathrm{~mm}^{2}$ for the individual MFL and EC scans.

Using Dempster-Shafer fusion, the possible outcomes (frame of discernment) for a particular location on the test specimens considered were $\theta=$ (defect, no defect). The results of fusing the MFL and EC data with the Dempster-Shafer technique are shown in Figure 7(b), where for the white regions there was $84 \%$ evidence that there was no defect at these positions with a plausibility of $100 \%$ supporting this conclusion. Similarly, the red areas mark defective regions with $84 \%$ evidence and $100 \%$ plausibility. The blue lines indicate regions where a defect had been detected with only one method but not with the other. Hence, after normalisation this yielded an evidential interval of [0.375; 0.625], i.e. with an evidence of $37.5 \%$ and a plausibility of $62.5 \%$ there was a defect present. The ignorance was $25 \%$ and the disbelief $37.5 \%$, as there was no information about which one of the two results was erroneous. Calculating the mean square error between the indicated and known defect positions yielded a figure of $973 \mathrm{~mm}^{2}$, again an improvement over the values of 1620 and $1940 \mathrm{~mm}^{2}$ for the individual MFL and EC scans. 

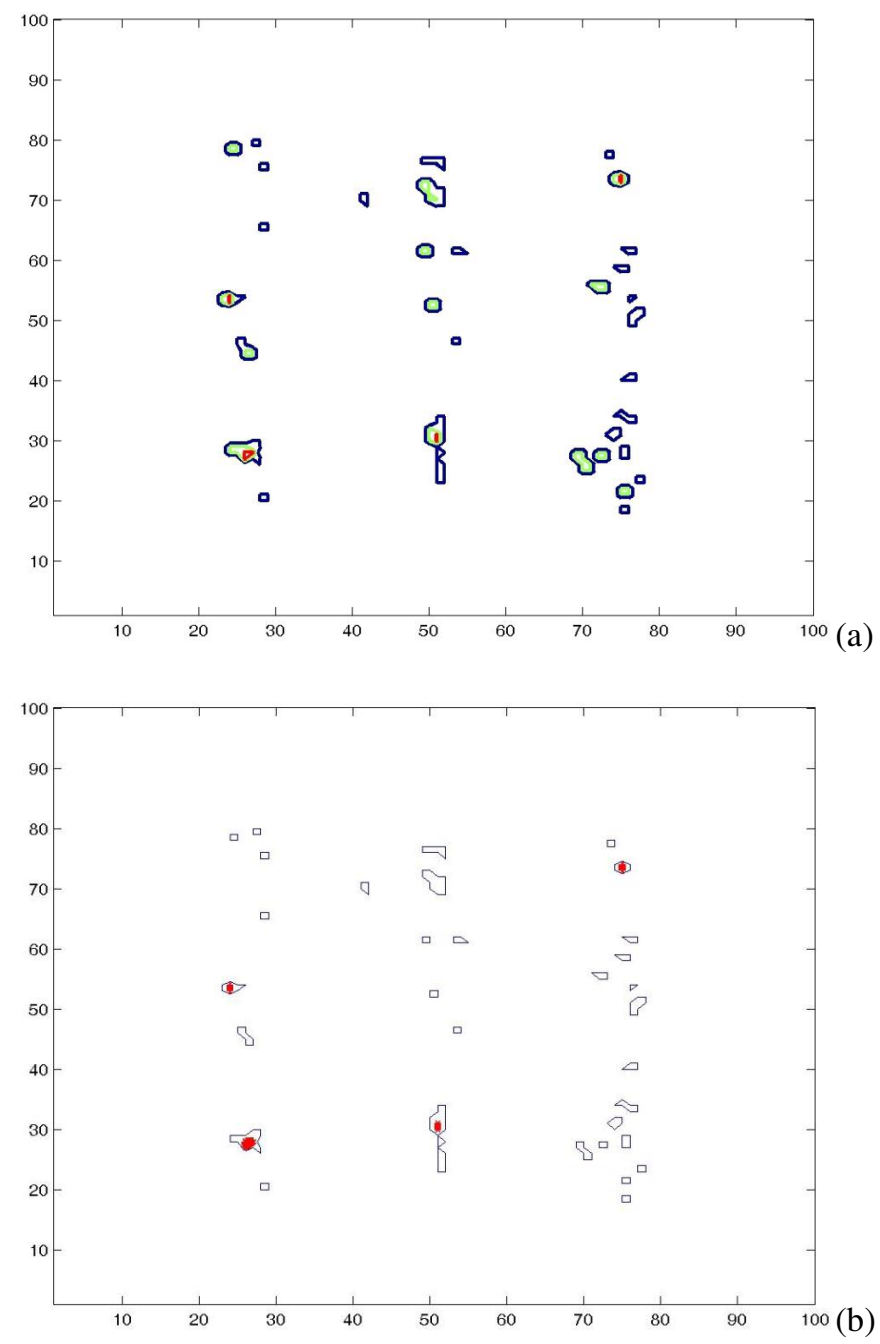

Figure 7: Data fusion for EC and MFL scans, using (a) Bayesian probabilistic and (b)Dempster-Shafer evidence theories

\section{DISCUSSION AND CONCLUSIONS}

Ultrasonic, MFL and EC-inspection offer different advantages depending on the particular application and the material under test, and with the aid of simple mathematical algorithms, a more complete assessment of the specimen can be achieved by fusing the different data sets. Shallow pits and thickness loss are easier to detect with the ultrasonic or MFL methods, while the EC sensor shows superior performance for small deep defects and cracks. As the $5 \mathrm{~mm}$ x $10 \mathrm{~mm}$ sensor head of the current EC-probe is flat, it is not suitable for curved surfaces and pipes, but can be applied to conducting non-ferromagnetic materials, such as aluminum. Depending on whether the critical flaw size is smaller than $2.6 \mathrm{~mm}$, in which case a defect could remain undetected by passing the Hall linear array precisely between two elements, MFL inspection is thirteen times faster compared to the EC-method, where the effective area covered by a single scan is just $1.5 \mathrm{~mm}$ wide. Even though EC-testing emerges as the most robust NDE technique, comparing the results of the two scans in Fig. 6 shows that successful qualitative detection of the machined defects is possible with both methods. An improved result however, is obtained by fusing the data. For both MFL and EC inspection an initial calibration of the payload on a sample defect is required prior to autonomous operation. A PoD for True Positive hits of $100 \%$ can be achieved when the sensor platforms are calibrated on a defect that is significantly smaller than the critical ones. Despite the size limitation, collectively the robotic sensor platforms possess a full set of NDE inspection capabilities and can be configured to suit the demands of different applications, environments and materials. A size and weight reduction of a factor of three compared to systems that have been previously appeared in literature has been achieved [5], [6], [36], [37], while versatility and complete autonomy without trade-off in the performance of various NDE payloads has been attained.

A second generation of inspection vehicles with a fundamental increase of on-board computing power and communication bandwidth is currently under development. Work is underway to integrate a visual inspection payload in the form of a miniature camera that can be used for both visual NDE and robot navigation. An automatic weld tracking algorithm is under development that will provide the sensor platforms with greater context awareness and can be used as an input signal for the on-board control system to allow the robot to accurately follow a weld. The camera interfaces directly with the on-board processor for embedded image processing.

Miniaturization is a key issue for these remote sensing agents as well as a refinement of the NDE circuitry and sensors. With enhanced circuitry and coils for eddy current inspection, defect quantification and detection of cracks smaller than $300 \mu \mathrm{m}$, which are typical for pressure vessels in nuclear power plants, is realistic. Furthermore, with a shorter distance between wheels, a higher magnetic field is created for MFL inspection, and with smaller size additional application areas like turbine blades or boiler tubes are established.

Future work will also consider separating the ultrasonic pitchcatch transducers onto different platforms allowing for long range ultrasonic transmission, paving the way for rapid scanning and the application of topographical algorithms for defect sizing and imaging.

\section{ACKNOWLEDGMENT}

This research received funding from the Engineering and Physical Sciences Research Council (EPSRC) and forms part of the core research program within the Research Centre for NDE, in the UK.

\section{REFERENCES}

[1] G. Caprari, K. Arras, and R. Siegwart, "The autonomous miniature robot Alice: from prototypes to applications," Intelligent Robots and Systems, 2000.(IROS 2000). Proceedings. 2000 IEEE/RSJ International Conference on, vol. 1, 2000.

[2] E. Petriu, T. Whalen, R. Abielmona, and A. Stewart, "Robotic sensor agents: a new generation of intelligent agents for complex environment monitoring," Instrumentation \& Measurement Magazine, IEEE, vol. 7, pp. 46-51, 2004.

[3] A. Drenner, M. LaPoint, I. Burt, K. Cannon, C. Hays, A. Kottas, and N. Papanikolopoulos, "Increasing the Scout's effectiveness 
through local sensing and ruggedization," Robotics and Automation, 2004. Proceedings. ICRA'04. 2004 IEEE International Conference on, vol. 2, 2004.

[4] A. Carvalho, I. Silva, J. Rebello, R. Carneval, and J. Farias, "Inspection of ship hulls using automated ultrasonic inspection," Insight-Non-Destructive Testing and Condition Monitoring, vol. 47, pp. 744-747, 2005.

[5] X. Song, X. Wu, and Y. Kang, "An inspection robot for boiler tube using magnetic flux leakage and ultrasonic methods," Insight-NonDestructive Testing and Condition Monitoring, vol. 46, pp. 275278, 2004.

[6] B. Blakeley, C. Emmanouilidis, and K. Hrissagis, "Above-ground storage tank inspection using the 'Robot Inspector'," Insight-NonDestructive Testing and Condition Monitoring, vol. 47, pp. 705708, 2005.

[7] N. Correll and A. Martinoli, "Collective Inspection of Regular Structures using a Swarm of Miniature Robots," Experimental Robotics IX: The 9th International Symposium on Experimental Robotics, 2006.

[8] C. Menon, M. Murphy, and M. Sitti, "Gecko Inspired Surface Climbing Robots," Robotics and Biomimetics, 2004. ROBIO 2004. IEEE International Conference on, pp. 431-436, 2004.

[9] K. Daltorio, S. Gorb, A. Peressadko, A. Horchler, R. Ritzmann, and R. Quinn, "A Robot that Climbs Walls using Micro-structured Polymer Feet," Climbing And Walking Robots: Proceedings of the 8th International Conference on Climbing and Walking Robots and the Support Technologies for Mobile Machines (CLAWAR 2005), 2006.

[10] H. Choset, "Coverage for robotics-A survey of recent results," Annals of Mathematics and Artificial Intelligence, vol. 31, pp. 113-126, 2001.

[11] J. Borenstein and L. Feng, "Correction of systematic odometry errors in mobile robots," Intelligent Robots and Systems 95.'Human Robot Interaction and Cooperative Robots', Proceedings. 1995 IEEE/RSJ International Conference on, vol. 3, 1995

[12] A. Smith, H. Balakrishnan, M. Goraczko, and N. Priyantha, "Tracking moving devices with the cricket location system," Proceedings of the 2nd international conference on Mobile systems, applications, and services, pp. 190-202, 2004.

[13] J. Borenstein, H. R. Everett, and L. Feng, Navigating Mobile Robots: Systems and Techniques: AK Peters, Ltd. Natick, MA, USA, 1996.

[14] G. Welch and G. Bishop, "An Introduction to the Kalman Filter," University of North Carolina at Chapel Hill, Chapel Hill, NC, 1995.

[15] D. Kim, L. Udpa, and S. Udpa, "Remote field eddy current testing for detection of stress corrosion cracks in gas transmission pipelines," Materials Letters, vol. 58, pp. 2102-2104, 2004.

[16] N. Yusa, Z. Chen, K. Miya, T. Uchimoto, and T. Takagi, "Largescale parallel computation for the reconstruction of natural stress corrosion cracks from eddy current testing signals," $N D T \& E$ international, vol. 36, pp. 449-459, 2003.

[17] G. Park and E. Park, "Improvement of the sensor system in magnetic flux leakage-typenondestructive testing (NDT)," Magnetics, IEEE Transactions on, vol. 38, pp. 1277-1280, 2002.

[18] C. Mandache and L. Clapham, "A model for magnetic flux leakage signal predictions," Journal Of Applied Physics, vol. 36, pp. 2427 2431, 2003.

[19] G. Wei and C. Jianxin, "A transducer made up of fluxgate sensors for testing wire ropedefects," Instrumentation and Measurement, IEEE Transactions on, vol. 51, pp. 120-124, 2002.
[20] R. Halmshaw, Non-Destructive Testing, 2 ed. London: Butterworth-Heinemann Ltd, 1991.

[21] COMSOL_Reaction_Engineering_Lab, "COMSOL," 3.4 ed: The COMSOL Group, 2008.

[22] R. Farlow and G. Hayward, "Real-time ultrasonic techniques suitable for implementing non-contact NDT systems employing piezoceramic composite transducers," Insight(Northampton), vol. 36, pp. 926-935, 1994.

[23] S. Kelly, R. Farlow, and G. Hayward, "Applications of through-air ultrasound for rapid NDE scanning inthe aerospace industry," Ultrasonics, Ferroelectrics and Frequency Control, IEEE Transactions on, vol. 43, pp. 581-591, 1996.

[24] S. P. Kelly, G. Hayward, T. E. G. Alvarez-Arenas, and M. Babcock, "Characterization and assessment of an integrated matching layer for air-coupled ultrasonic applications," Ultrasonics, Ferroelectrics and Frequency Control, IEEE Transactions on, vol. 51, pp. 1314-1323, 2004

[25] G. Georgiou, "PoD curves, their derivation, applications and limitations," Insight-Non-Destructive Testing and Condition Monitoring, vol. 49, pp. 409-414, 2007.

[26] S. Thrun, W. Burgard, and D. Fox, Probabilistic Robotics (Intelligent Robotics and Autonomous Agents): MIT press, Cambridge, Massachusetts, USA, 2005.

[27] V. Kaftandjian, Y. Zhu, O. Dupuis, and D. Babot, "The combined use of the evidence theory and fuzzy logic for improving multimodal nondestructive testing systems," Instrumentation and Measurement, IEEE Transactions on, vol. 54, pp. 1968-1977, 2005.

[28] O. Duran, K. Althoefer, and L. Seneviratne, "Automated Pipe Defect Detection and Categorization Using Camera/Laser-Based Profiler and Artificial Neural Network," Automation Science and Engineering, IEEE Transactions on [see also Robotics and Automation, IEEE Transactions on], vol. 4, pp. 118-126, 2007.

[29] A. Dogandzic and B. Zhang, "Bayesian NDE Defect Signal Analysis," IEEE Transactions On Signal Processing, vol. 55, p. 372, 2007.

[30] T. Chen and V. Venkataramanan, "Dempster-Shafer Theory for Intrusion Detection in Ad Hoc Networks," IEEE Internet Computing, pp. 35-41, 2005.

[31] Z. Liu, D. Forsyth, J. Komorowski, K. Hanasaki, and T. Kirubarajan, "Survey: State of the Art in NDE Data Fusion Techniques," Instrumentation and Measurement, IEEE Transactions on, vol. 56, pp. 2435-2451, 2007.

[32] D. MacKay, Information Theory, Inference and Learning Algorithms: Cambridge University Press, 2003.

[33] G. Shafer, A mathematical theory of evidence: Princeton University Press Princeton, NJ, 1976.

[34] A. Dempster and P. File, "Upper and Lower Probabilities Induced by a Multivalued Mapping," Classic Works on the Dempstershafer Theory of Belief Functions, 2008.

[35] K. Sentz and S. Ferson, "Combination of evidence in DempsterShafer theory," Report No. SAND2002, vol. 835, 2002.

[36] G. La Rosa, M. Messina, G. Muscato, and R. Sinatra, "A low-cost lightweight climbing robot for the inspection of vertical surfaces," Mechatronics, vol. 12, pp. 71-96, 2002.

[37] T. Sattar, H. Rodriguez, J. Shang, and B. Bridge, "Automated NOT of Floating Production Storage Oil Tanks with a Swimming and Climbing Robot," Climbing And Walking Robots: Proceedings of the 8th International Conference on Climbing and Walking Robots and the Support Technologies for Mobile Machines (CLAWAR 2005), 2006. 